\title{
De la Covid-19 vers une métropole résiliente : l'exemple du Grand Nancy
}

Charlotte Copplet, Martine Batt, André Rossinot et Jane-Laure Danan

\section{OpenEdition}

1 Journals

Édition électronique

URL : http://journals.openedition.org/rfst/836

DOI : $10.4000 /$ rfst.836

ISSN : 2492-3672

Éditeur

Espaces et SOciétés (UMR 6590)

Référence électronique

Charlotte Copplet, Martine Batt, André Rossinot et Jane-Laure Danan, « De la Covid-19 vers une métropole résiliente : l'exemple du Grand Nancy », Revue francophone sur la santé et les territoires [En ligne], Pandémie, crises et perspectives : lectures territoriales de la Covid-19, mis en ligne le 18 février 2021, consulté le 06 avril 2021. URL : http://journals.openedition.org/rfst/836 ; DOI : https://doi.org/ $10.4000 /$ rfst.836

Ce document a été généré automatiquement le 6 avril 2021.

\section{(c) (i) (8)}

La Revue francophone sur la santé et les territoires est mise à disposition selon les termes de la Licence Creative Commons Attribution - Pas d'Utilisation Commerciale - Partage dans les Mêmes Conditions 4.0 International. 


\title{
De la Covid-19 vers une métropole résiliente : l'exemple du Grand Nancy
}

\author{
Charlotte Copplet, Martine Batt, André Rossinot et Jane-Laure Danan
}

\section{Introduction}

1 Cet écrit s'inscrit dans le cadre d'un stage à la Métropole du Grand Nancy en tant qu'étudiante en master de psychologie clinique à l'Université de Lorraine. Les conditions de réalisation de ce stage ont été bouleversées par le contexte de pandémie et la situation de confinement. Il a été alors conseillé de réaliser une étude sur le fonctionnement métropolitain dans la gestion de crise dont la finalité est d'évaluer les risques psychosociaux engendrés par la situation de confinement avec l'objectif d'orienter les actions concrètes à mettre en place pour garantir au mieux le bien-être de la population grand-nancéienne.

2 La pandémie de Covid-19 représente la crise sanitaire la plus grave depuis un siècle, une crise aux multiples implications sanitaires, sociales et sociétales, d'autant plus dans la région Grand-Est qui fut l'une des plus durement touchées par l'épidémie. La population est restée confinée pendant près de deux mois nécessitant une adaptation des collectivités territoriales à la préparation de la sortie du confinement. Le retour progressif à un mode de vie libéré de tout ou partie des restrictions de liberté nécessite une préparation. La situation de confinement est une expérience exceptionnelle individuelle et collective qui présente des risques d'impacts psychologiques pouvant être délétères. De plus, selon une étude publiée en mars 2020 (Brooks, S. et al), une période de confinement supérieure à 10 jours peut entraîner des symptômes de stress post-traumatique. Pour faire face à cette situation inédite, il a semblé nécessaire de mettre en place des stratégies collectives et individuelles pour renforcer la résilience, à savoir la "capacité à s'adapter et à rebondir" (Molenda, CN2R, 2020). La situation de confinement nous oblige à repenser nos habitudes aussi bien dans la vie quotidienne 
qu'au travail, mais également à repenser nos organisations et nos projections. Jack Shenker (Courrier International, 2020) s'est intéressé à l'impact des crises sanitaires sur les métropoles et se demande comment concilier densification urbaine et respect des règles de distanciation sociale.

Tout comme lui, beaucoup d'auteurs et de chercheurs s'appuient sur l'aspect historique des différentes crises subies dans le monde pour mettre en avant la capacité de " résilience urbaine ", à savoir la capacité d'une ville à se réinventer. De nos jours, cette compétence pourrait se traduire non seulement par un réaménagement des infrastructures urbaines, mais également par un développement massif des infrastructures numériques et du télétravail (Harris, K., 2020).

4 La notion de résilience est définie par le domaine de la physique comme la résistance des matériaux à des chocs élevés et celui de l'informatique comme la capacité à continuer de fonctionner malgré des anomalies. Puis la psychologie s'est emparée de cette notion pour définir la "capacité d'une personne ou d'un groupe à se développer bien, à continuer à se projeter dans l'avenir en dépit d'évènements déstabilisants, de conditions de vie difficiles, de traumatismes sévères » selon Manciaux, Lecomte et Cyrulnik (2001). La notion de résilience représente un processus dynamique constitué par des facteurs de risque et des facteurs de protection qui vont déterminer la capacité d'un individu à se ressaisir, à élaborer puis à continuer de se développer malgré le risque de désorganisation psychique. La résilience implique également une forme de valeur ajoutée, une remobilisation des forces chez le sujet qui ressort plus fort et plus créatif.

5 La résilience urbaine représente la capacité d'une ville à absorber une perturbation puis à récupérer ses fonctions par la suite selon Lhomme \& al (2010). Les auteurs soulèvent comme facteurs d'amélioration de la résilience urbaine, la limitation de la perturbation du système par une meilleure capacité de résistance et d'absorption, ainsi qu'une stratégie d'accélération du retour à la normale par une gestion optimisée des moyens et des ressources, et une bonne accessibilité. Campanella (2006) situe la résilience urbaine dans le domaine de la gestion des risques et la définit comme «la capacité d'une ville à faire face à un événement destructeur tout en minimisant ses dommages ». Parmi les facteurs d'influence de cette reconstruction, Campanella relève notamment l'importance des réseaux techniques et des services urbains.

6 Cet écrit s'appuie alors sur la conjoncture entre résilience urbaine et résilience individuelle puisque les stratégies de résilience mises en place par la Métropole visent en premier lieu à promouvoir et accompagner la résilience individuelle et collective de sa population. Dans cette perspective, transformer la ville et ses services urbains, c'est s'appuyer sur les difficultés rencontrées pendant cette période de crise sanitaire pour en faire des leviers pour la ville de demain.

7 Le confinement représente une période d'isolement et la sortie du confinement représente une étape transitoire vers un retour progressif à un mode de vie libéré de toute ou parties des restrictions de liberté. L'objectif a été de préparer au mieux la sortie du confinement dans le respect des règles sanitaires. La problématique investie dans cette étude est comment préparer la convalescence sociale et sociétale sur le long terme grâce à une approche globale de la résilience métropolitaine?

8 En effet, cette situation risque d'induire de multiples séquelles dans la société, tant pour les individus que pour de nombreux acteurs économiques. Cet état pourrait accentuer les inégalités en santé. C'est pourquoi la Métropole du Grand Nancy a souhaité porter un plan de transition vers ce «jour d'après ». Au-delà d'un plan 
strictement sanitaire de sortie de confinement, ce projet s'entend comme une approche globale de résilience.

\section{La mission santé et bien-être de la Métropole}

La Métropole du Grand Nancy est composée de 20 communes et regroupe 256769 habitants, dont 49000 étudiants. Sa densité de population s'élève à 1804,4 habitants/ $\mathrm{km}^{2}$ (INSEE - 2017). L'agglomération grand nancéienne comporte 8 quartiers prioritaires de la politique de la ville (QPV) répartis sur 9 communes (cf. figure 1). Les QPV ont été établis par la loi de programmation pour la Ville et la cohésion urbaine du 21 février 2014 dans le but de réduire les écarts de développement au sein des villes. La population totale de ces 8 quartiers s'élève à 32650 habitants (RFL 2011). Selon l'agence de développement des territoires Nancy Sud Lorraine (SCALEN), les QPV de la Métropole du Grand Nancy présentent un taux de pauvreté de $44 \%$.

Figure 1. Quartiers Prioritaires de la politique de la Ville, Métropole du Grand Nancy

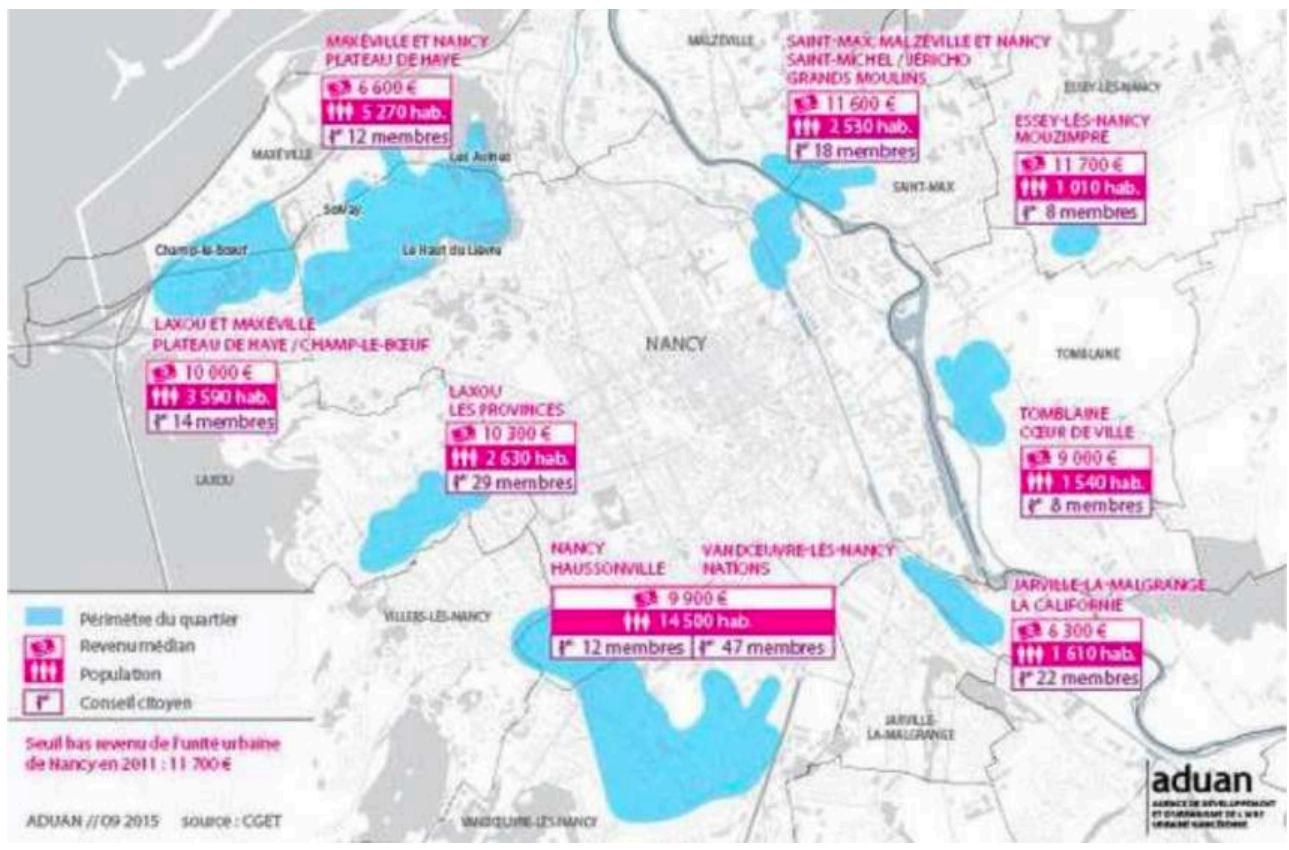

Source : Métropole du Grand Nancy. (2015). Contrat de ville du Grand Nancy 2015-2020. Reproduction autorisée par la Mission Santé Bien-Être de la Métropole du Grand Nancy. Consultable en ligne https://www.grandnancy.eu/fileadmin/fichiers/LA_METROPOLE/Democratie_participative/ 2018_06_12_contrat_de_ville.pdf

10 La promotion de la santé et la réduction des inégalités territoriales constituent un axe majeur du projet métropolitain. Pionnière du Réseau des Villes-Santé de l'Organisation Mondiale de la Santé, la Métropole du Grand Nancy est engagée depuis plusieurs années dans la promotion de la santé et du bien-être avec des attentes particulières: la réduction des inégalités territoriales, environnementales et sociales, ainsi que l'autonomie des grands nancéiens en matière de santé. Son rôle est de coordonner et fédérer les dynamiques locales entre acteurs et partenaires sur le terrain, ainsi que de leur offrir une visibilité par le biais d'une information régulière. La santé constitue un enjeu majeur tant du point de vue de la qualité de vie des habitants que de l'attractivité du territoire. La concrétisation de ce projet a donné lieu à un premier Contrat Local de 
Santé (CLS) intercommunal sur la période 2013-2017 signé par la Métropole du Grand Nancy, l'Agence Régionale de Santé et la Préfecture de Meurthe-et-Moselle. Le CLS a pour objectif l'articulation, la coordination et la mise en cohérence des politiques de santé et des dynamiques locales sur les questions de santé tout en tenant compte des besoins et des leviers existants dans les territoires. Le Contrat Local de Santé intercommunal de deuxième génération 2019-2023 est composé de 52 actions réparties en 5 axes concernant l'environnement, l'accès aux soins, la qualité de vie, la pratique physique et le comportement alimentaire, ainsi que la prévention des risques dans le domaine des addictions. Les orientations présentées dans le CLS 2019-2023 résultent d'une démarche participative impliquant une consultation citoyenne à l'occasion de laquelle le public métropolitain a sélectionné puis classé par ordre de priorité les 5 axes d'action mentionnés ci-dessus. La Métropole dispose également d'un rôle de prévention et d'information du grand public, notamment dans le cadre de la lutte contre les fake news. Dans le cadre du Contrat Local de Santé intercommunal 2ème génération 2019-2023, la Métropole a accompagné et soutenu les professionnels de santé pendant la crise de la Covid-19 avec la mise en place de diverses plateformes d'écoute médicopsychologiques et de dispositifs de transport adaptés. Pour faire face à ce contexte de crise, de nombreuses actions ont pu être réalisées de manière collaborative avec l'appui métropolitain, autant en termes d'accompagnement qu'en termes logistique : la cellule de réflexion après confinement, le dispositif «Prévention Conséquences Confinement", la dotation de matériel aux soignants, le déploiement d'outils d'accompagnement et de soutien tels que les plateformes d'écoute, un dispositif de transport adapté pour le personnel hospitalier, une commande groupée de masque pour les communes de la Métropole, et la lutte contre les fake news.

11 Le caractère tout à fait exceptionnel de la crise et les incertitudes qui subsistent sur les modes de transmission du virus ne doivent pas dispenser d'être, au niveau local, dans une posture de vigilance à l'égard de la population, d'accompagnement de celle-ci et d'initiatives complémentaires au cadre fixé par l'État. Ainsi, les risques sanitaires liés à la Covid-19 ont nécessité une adaptation des collectivités territoriales, et la Métropole a mis en place des mesures afin de doter l'ensemble de ses 20 communes de ressources pour préparer au mieux la sortie du confinement, les aider à se réorganiser. Les schémas ci-dessous présentent une population grand-nancéienne grandissante, ainsi qu'une importante concentration d'habitants sur certaines zones de la métropole. Cette répartition impose une vigilance particulière pour garantir la sécurité des citoyens (cf. figure 2). 
Figure 2. Nombre moyen d'habitants à l'hectare urbanisé en 2016 et taux d'évolution moyenne annuelle de la population entre 2011 et 2016

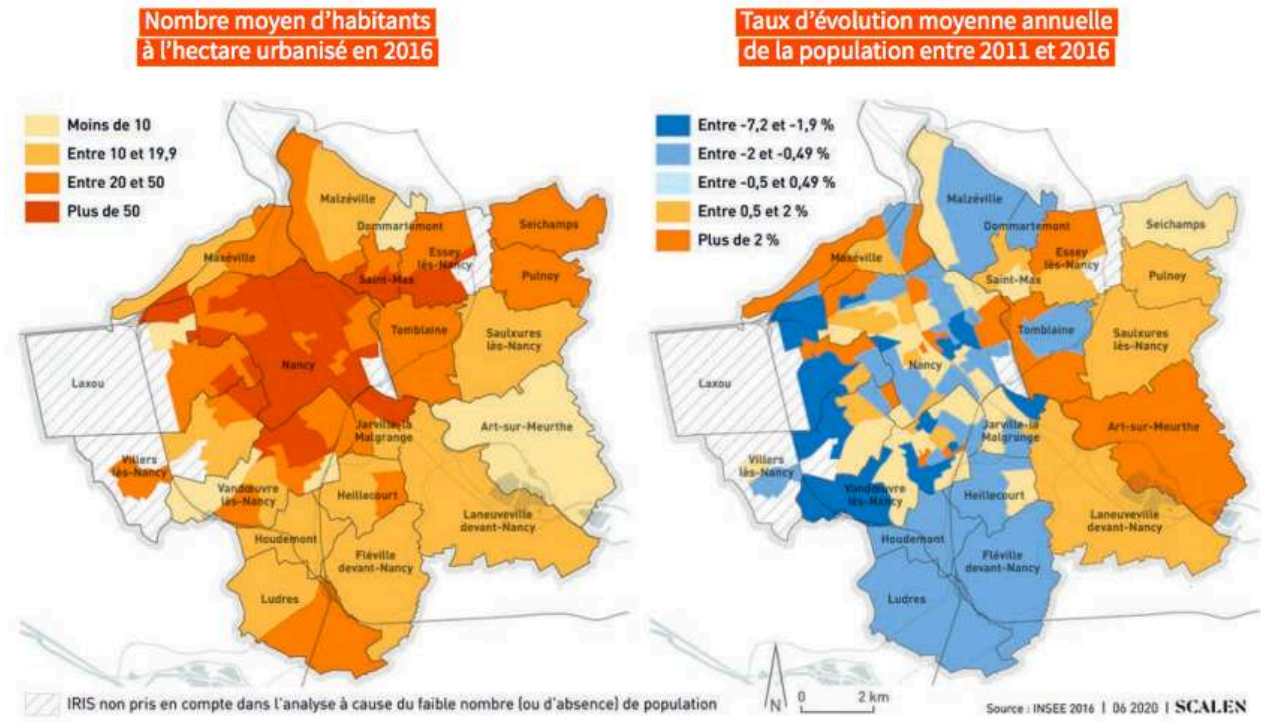

Source : Agence SCALEN. (2020). Atlas Santé Bien-Être de la Métropole du Grand Nancy.

Reproduction autorisée par la mission santé bien-être de la Métropole du Grand Nancy. Consultable en ligne : https://www.grandnancy.eu/fileadmin/fichiers/VIVRE_ET_HABITER/Sante/Atlas_Sante.pdf

\section{Apports de la littérature}

12 Ce corpus théorique porte sur les répercussions psychosociales du confinement et sur les notions de résilience individuelle et de résilience urbaine. Nous avons tout d'abord recherché diverses associations de mots clés sur le thème «impact psychosocial et confinement » ainsi que sur les notions de "résilience » et " résilience urbaine », dans les bases de données Cairn, Google Scholar et Science Direct. L'étude de Brooks \& al (2020) publiée dans le Lancet a servi de base à notre réflexion sur les répercussions psychosociales provoquées par la situation de confinement. La majorité de nos ressources bibliographiques scientifiques datent de 2020 puisqu'elles portent sur un contexte sanitaire inédit. Le peu de travaux disponibles sur les répercussions psychosociales de la situation de confinement se concentrent généralement sur les patients contaminés. De ce fait, nous avons choisi de nous appuyer subsidiairement sur certains articles de presse qui recueillent le point de vue de professionnels psychologues et urbanistes, en complément des articles scientifiques. Nous avons également choisi d'investir les recommandations de la Haute Autorité de Santé et les rapports officiels. Enfin, nous nous sommes concentrés sur trois domaines, en recherchant dans les mêmes bases de données différentes équations de recherche sur les thèmes suivants : "confinement et travail ", " confinement et santé mentale ", "confinement et mobilité », "impact psychologique et confinement", «post confinement». La finalité de ce corpus théorique est d'appuyer cette recherche, notamment en ce qui concerne les conséquences psychosociales générées par la situation de confinement. L'idée étant d'apporter un cadrage théorique aux actions concrètes mises en place. 
13 Nous avons envisagé d'un point de vue théorique certains items correspondant aux actions menées : le retour au travail, les conditions sanitaires, le rôle des managers, l'accompagnement en santé mentale ainsi que les transports, la mobilité et l'espace public.

\section{Sur le retour au travail}

\section{Sur les conditions sanitaires}

14 La priorité est d'assurer la sécurité des travailleurs en continuant de favoriser le télétravail autant que possible et de mettre en œuvre les conditions permettant le respect des gestes barrières et des règles de distanciation sociale. Il est essentiel que le retour au travail se réalise de façon progressive. En effet, le Center for the Study of Traumatic Stress, rappelle que l'isolement prolongé entraîne une perte de routine quotidienne. Il faut donc prendre en compte le fait que le retour au travail et aux routines sociales nécessite plusieurs semaines, voire plusieurs mois. Ainsi, chaque individu et chaque institution doit être en mesure de s'adapter à la situation et de repenser son organisation et ses objectifs de manière progressive. Favoriser le recours au télétravail permettrait d'éviter un nombre trop important d'agents présents simultanément sur le lieu de travail et de limiter les recours aux transports publics. Pour autant, il faut être attentif au fait de ne pas accentuer le sentiment d'isolement social (C. Tourette-Turgis, 2020). En effet, selon l'étude de Tissandier et MarianiRousset (2019), si le télétravail semble présenter de nombreux points positifs (moins de stress et de fatigue, meilleurs rapports professionnels et familiaux), il peut également engendrer des conséquences négatives pour certains travailleurs (potentiel manque de régulation, source d'envahissement de la vie privée).

\section{Sur le rôle des managers d'équipe}

Adrien Chignard, psychologue du travail et Catherine Tourette-Turgis, psychologue clinicienne spécialiste du retour au travail des patients atteints de maladies chroniques et de cancers ont été interviewés sur les modalités de retour au travail. Catherine Tourette-Turgis conseille aux managers d'informer les salariés, en toute transparence, de la situation de l'entreprise car ces derniers peuvent être angoissés par d'éventuelles réorganisations ou par une surcharge de travail impossible à assumer (c. TouretteTurgis). Ensuite, Adrien Chignard suggère de réunir les équipes, dès le premier jour de travail, dans l'objectif de créer une expérience collective autour de l'évènement vécu, permettant ainsi de refonder un esprit d'équipe bénéfique pour la productivité des salariés. À cette occasion, il conseille également d'inviter chaque salarié à se prononcer sur les aspects positifs et négatifs de l'organisation du travail pendant le confinement. Le but est de réduire le stress en permettant à chacun d'exposer ses difficultés et d'augmenter la motivation en impliquant les salariés. Catherine Tourette-Turgis met en garde contre le sentiment d'isolement social, notamment si le contact a été rompu pendant le confinement. Sur la question du chômage partiel, elle met en avant le risque de rupture du sentiment d'appartenance. Pour faire lien avec les propos de A. Chignard, un employé en situation d'isolement avec qui le contact a été rompu pendant le confinement peut développer une rupture du sentiment d'appartenance à l'entreprise, générant une souffrance morale, un risque de désaffiliation et donc une perte de motivation. Sylvie Tordjman, pédopsychiatre au centre hospitalier de Rennes, défend 
également le maintien des liens d'équipe comme un levier renforçant le sentiment d'appartenance. Son équipe est restée en lien par le biais de réunions téléphoniques hebdomadaires, favorisant une cohésion de groupe autour d'une expérience commune et permettant une entraide pour appréhender ce nouveau fonctionnement de travail.

\section{Sur l'accompagnement en santé mentale} traumatique comme une hypervigilance. L'état de stress-post traumatique est défini comme "une réponse différée ou prolongée à une situation ou à un évènement stressant (de façon directe ou indirecte), exceptionnellement menaçant ou catastrophique et qui provoquerait des symptômes évidents de détresse chez la plupart des individus»(CIM-10, 2008). Ce risque est accentué par l'exposition aux médias. Marianne Kedia, psychologue spécialiste du trauma, nous explique que «les chaînes d'info fonctionnent comme un cerveau traumatisé (...) il reçoit une charge émotionnelle très forte renforcée par l'effet hypnotique des images. ». Il a été démontré, et rappelé par l'OMS, que les symptômes anxieux générés par la situation de confinement augmentaient avec le manque d'informations claires et fiables généré par la quantité massive d'informations, parfois contradictoires, à notre disposition. L'étude du Dr Haesebaert et du Pr Franck (CH Le Vinatier) nous rappelle que le niveau de bien-être des individus dépend du niveau d'accord avec la mesure, de la satisfaction de l'information communiquée et de l'accès aux produits de première nécessité et de protection. De même, diverses études ont démontré que l'adhésion à la mesure et la façon d'envisager le confinement rendaient le stress et la frustration plus facilement supportables. De ce fait, proposer une sensibilisation aux médias et à la recherche d'information peut représenter un moyen efficace pour lutter contre l'anxiété et l'état d'alerte générés par ces derniers. En effet, Sylvie Molenda (CN2R) nous rappelle qu'il est nécessaire de se tenir informé par le biais de sites officiels, comme Santé Publique France, et de bannir les médias qui emploient des moyens pour dramatiser les situations. Enfin, il est important de renforcer le sentiment d'altruisme et de solidarité généré par le fait de protéger les autres et de sauver des vies. Ainsi, l'étude publiée dans le Lancet (Brooks, S. et al, 2020) préconise de promouvoir une communication plutôt centrée sur l'altruisme que sur l'obsession, et de remercier, encourager les individus en situation de confinement afin de renforcer l'adhésion à la mesure.

\section{Sur la mobilité, les transports et l'espace public}

18 Concernant l'espace public, divers experts, dont l'urbaniste new yorkais Jens Aert, proposent de préserver notre "santé urbaine » par le biais d'une réorganisation des 
villes laissant place à davantage de pistes cyclables et de zones piétonnes (Courrier International, 2020).

Pour autant, il est important de noter que la situation sanitaire actuelle qui appelle à privilégier les infrastructures numériques et les procédures dématérialisées, vient profondément creuser la fracture numérique et les inégalités d'accès aux services publics et au télétravail. G. F. Dumont, dans son article "Covid-19: la fin de la géographie de l'hypermobilité ?" nous rappelle que cette pandémie nous oblige à repenser l'aménagement du territoire, notamment en accélérant l'accès à l'égalité numérique afin de permettre à tout élève/étudiant de pouvoir poursuivre ses enseignements à distance, et à tout travailleur de pouvoir continuer son activité en télétravail. En effet, Dumont estime qu'une partie des travailleurs est amenée à continuer son activité en télétravail, et ce pour une durée indéterminée.

\section{Matériel et méthode}

Pour mener à bien ce travail, la Métropole a constitué une cellule de réflexion pluridisciplinaire. Nous avons analysé les demandes adressées aux experts de la cellule selon des modalités de recueil qui figurent ci-après (cf. figure 3). Puis nous avons confronté ces éléments à la littérature. La figure 3 présente le tableau permettant le recueil des données des communes vers la cellule d'experts. Ce tableau permet, d'une part, de répertorier les diverses demandes émanant des communes ainsi que la nature du demandeur, et d'autre part, de conserver une traçabilité des réponses apportées par les experts. La figure 3 vise à mettre en avant la méthodologie de recueil. Le contenu anonymisé est donné à titre d'exemple.

Figure 3. Tableau d'analyse des demandes adressées aux experts de la cellule

\begin{tabular}{|c|c|c|c|c|c|c|}
\hline & \multicolumn{6}{|c|}{ Groupe d'experts - Accompagnement de laprès confinement } \\
\hline Date of I Idemannde & Demandeur & Naturuo de I I domande & Date dela rictonsse & Raporstour & Reponse & OBSERVATIONS \\
\hline 11/0532020 & mosisurer & 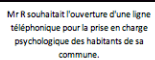 & $11 / 105 / 2020$ & Masaneo & 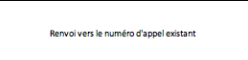 & \\
\hline $12 / 2552020$ & masamec & 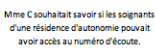 & 12/205/20200 & Mastme o & 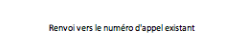 & 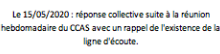 \\
\hline 2010552020 & masanec & 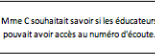 & 29195520200 & Madane D & 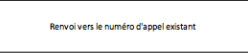 & 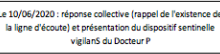 \\
\hline 1206520200 & madanec & 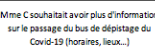 & 12/206/20200 & 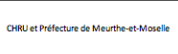 & 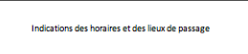 & \\
\hline 12/2060202020 & Maresect & 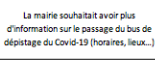 & 12/206/20200 & 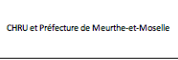 & 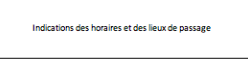 & \\
\hline 17/66520200 & masanen & 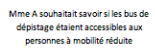 & $17 / 66520200$ & Mataseo & 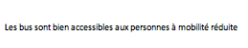 & \\
\hline
\end{tabular}

Source : document interne à la Métropole du Grand Nancy, non publié. Reproduction autorisée par la Mission Santé Bien-Être de la Métropole du Grand Nancy.

Pour établir une stratégie globale d'accompagnement des citoyens de manière complémentaire au cadre fixé par l'État, la Métropole du Grand Nancy a sollicité ses partenaires afin de constituer une cellule de réflexion. Cette Cellule pluridisciplinaire de Réflexion Avant sortie de Confinement (CRAC) est composée de 32 experts (médecins, épidémiologistes, hygiénistes, biologistes, pharmaciens, psychologues, sociologues, élus...) déjà partenaires de la Métropole du Grand Nancy, dans le cadre du Contrat Local de Santé intercommunal de deuxième génération 2019-2023. L'objectif de cette cellule a été de formuler des préconisations pour préparer au mieux la sortie du confinement. Elle s'est réunie à plusieurs reprises en visioconférence sous forme de 
groupes restreints, puis lors d'une séance plénière en date du 22 avril 2020. Ainsi, une liste de 16 préconisations a pu être établie. Ce matériel a constitué la base de la réflexion.

En parallèle de cette cellule, la littérature a été mobilisée, d'une part dans l'objectif d'évaluer les répercussions psychosociales impliquées par la situation de confinement et d'orienter les actions nécessaires à mettre en place pour accompagner les processus de résilience individuelle et collective. D'autre part, nous nous sommes intéressés à la notion de résilience urbaine qui correspond à la capacité d'une ville à se réinventer, et à concilier densification urbaine et respect des règles de distanciation sociale. Ainsi, la Métropole a réfléchi à la manière de réinventer les espaces, notamment grâce à un développement des infrastructures numériques et un réaménagement des infrastructures urbaines. Cette démarche s'appuie sur le postulat que le développement de la résilience urbaine constitue un pilier pour aider les citoyens à mobiliser et développer leurs propres capacités de résilience individuelle.

Ce travail a débuté pendant le confinement afin d'agir le plus précocement possible, mais également afin que cette cellule soit opérationnelle le 11 mai 2020, date officielle de la sortie du confinement, pour se constituer en dispositif à disposition des représentants de communes. Ce système de communication entre représentants des communes et experts de la cellule, à l'initiative de la Métropole du Grand Nancy, a permis une réactivité et une réponse au plus proche des besoins des habitants. À ce jour, la cellule a été sollicitée à plus de 40 reprises pour des demandes concernant le champ de l'accompagnement et le champ de la logistique.

L'ensemble de ces travaux s'est concrétisé sous la forme d'un plan de résilience métropolitaine. Ce plan, à vocation très opérationnelle, concerne l'accompagnement de la crise sanitaire, la cohésion de la société métropolitaine, le travail et les activités économiques, les mutations économiques et écologiques, la mobilité et l'espace public, et enfin, l'éducation, la culture, les sports, les loisirs et la santé. Ce dernier a également pour vocation d'aider les communes à mieux anticiper toutes éventuelles situations de crise à venir.

\section{Résultats et analyse}

\section{Les actions concrètes menées par la Métropole en temps de crise}

Par le biais de son plan de résilience métropolitaine, la Métropole du Grand Nancy a établi des plans d'action dans les domaines visés par la littérature comme des secteurs impactés de manière privilégiée par la crise sanitaire. Il s'agit des domaines suivants : l'accompagnement du retour au travail à travers son plan de reprise progressive, l'accompagnement en santé mentale à travers son dispositif « prévention conséquences confinement » et l'accompagnement de la mobilité et de la transformation de l'espace public à travers un réaménagement des infrastructures.

\section{Sur le retour au travail des agents}

Dans un premier temps, pour répondre à la demande de reprise progressive des agents, différentes organisations dont la Métropole ont mis au point des plans de reprise d'activité conciliant le souci de production efficace pour contribuer à la relance du 
territoire avec les enjeux sanitaires. Face à un contexte totalement inédit, nécessitant de concilier santé physique et psychique, et solidité économique, les institutions ont encore à réfléchir sur des stratégies permettant de garantir la santé globale des travailleurs. En effet, le télétravail est amené à se développer, mais nécessite de prendre en compte les potentiels inconvénients associés tels que la difficulté de travailler en équipe, le sentiment d'isolement social et le risque de rupture du sentiment d'appartenance soulevés par Catherine Tourette-Turgis. En appui sur les recommandations d'Adrien Chignard (2020), les institutions doivent s'appuyer sur leurs managers d'équipe pour conserver une proximité dans les échanges et garantir la continuité du sentiment d'appartenance et d'affiliation à l'équipe. De même, pour répondre au besoin de transformer nos collectifs de travail et notre culture d'entreprise, la Métropole souhaite développer un «club des employeurs du Grand Nancy " dans le but de partager notre expérience, de s'entraider et de renouveler ensemble notre culture territoriale sur le sujet. Ce projet vise à créer une expérience collective autour de l'évènement vécu et trouver des solutions ensemble pour refonder un esprit d'équipe bénéfique pour la productivité des salariés tel que suggéré par Adrien Chignard.

Pour faire face à une probable augmentation de la demande, le Médecin du Travail de la Métropole préconise une mutualisation des services de Prévention des différentes villes de la Métropole, ainsi que la mise en place d'une plateforme d'écoute médicopsychologique. De plus, il incite à développer la prise en charge existante menée par le réseau psychologues de ville, constitué des psychologues installées en libéral, grâce à un appel à la mobilisation.

De même, une commande groupée de masques pour chaque citoyen de la Métropole et de ses communes a été réalisée dans le but de garantir un retour au travail en toute sécurité. Et pour répondre aux problématiques d'approvisionnement en matériel de protection rencontrées par les soignants, un appel au don a été lancé en collaboration avec la Communauté Professionnelle Territoriale de Santé (CPTS). La CPTS a ainsi mis en place une plateforme de don et de distribution du matériel de protection.

Enfin, parce que les conséquences économiques de la crise sanitaire risquent également d'impacter les étudiants et la poursuite de leurs études, la Métropole se donne pour objectif d'accueillir une centaine de jeunes en alternance, ainsi qu'une centaine de jeunes en stage afin de les aider à concrétiser leur projet d'insertion professionnelle.

\section{Sur l'accompagnement en santé mentale}

30 Pendant la crise de la Covid-19, des réunions hebdomadaires avec les élus sociaux ont permis d'identifier les besoins et d'adapter les actions mises en place. Ces actions visent à mettre en place des solutions concrètes face aux divers risques d'impacts psychologiques délétères soulevés par Brooks \& al (2020). Il s'agit de prévenir les diverses conséquences psychologiques générées par la situation de confinement et l'isolement, mais également d'apporter un soutien aux personnes impactées par la maladie ainsi qu'aux personnes endeuillées.

31 Cette démarche a notamment permis la création du dispositif "prévention conséquences confinement " en collaboration avec la Plateforme Territoriale d'Appui (PTA) qui a assuré la formation de plus de 160 étudiants bénévoles afin de mettre en place diverses actions. Ces actions visent à prévenir les risques d'impacts 
psychologiques délétères provoqués par la situation de confinement, notamment à type post-traumatique, et également de prévenir les hospitalisations des personnes fragiles confinées (cf. figure 4). La figure 4 présente les divers partenaires impliqués dans le dispositif. Les flèches représentent les liens permanents et réciproques entre les porteurs du projet et les partenaires sur le terrain qui sont en contact direct avec le public. Des dispositifs d'accompagnement ont notamment été mis en place par le biais d'un contact téléphonique régulier avec les personnes isolées, les personnes en situation de handicap ou bien les personnes âgées vulnérables. Un dispositif de courses livrées directement à domicile par les commerçants ou par les bénévoles a également été instauré. Ont également vu le jour des plateformes à destination du grand public et des familles endeuillées, d'autres à destination des salariés de petites et moyennes entreprises. Un dispositif particulier d'écoute et de soutien a été mis en place pour les soignants en collaboration avec le Centre Psychothérapique de Nancy.

Figure 4. Dispositif Prévention Conséquences Confinement

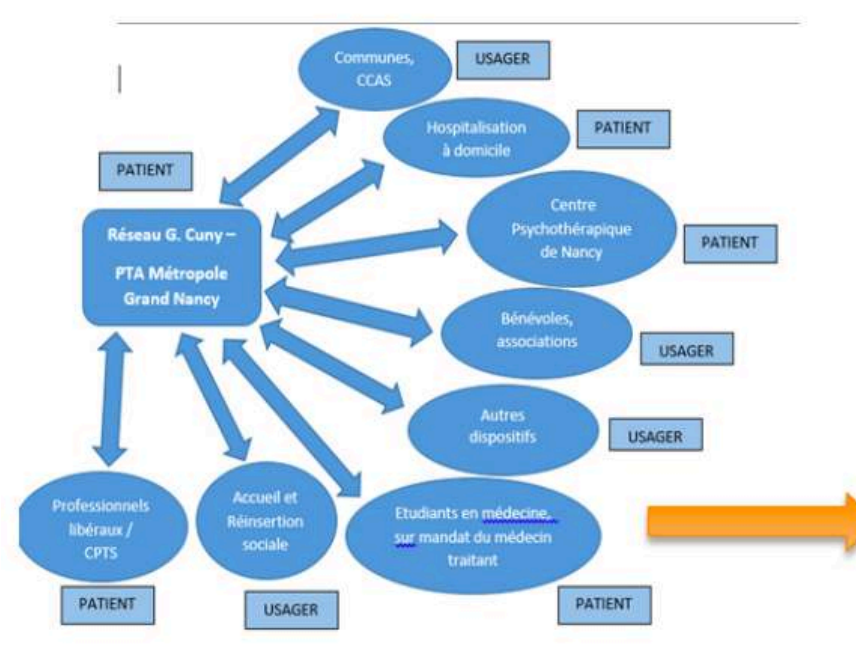
Circuit coordonné d'alerte et de partage d'informations utiles en fonction des missions et compétences de chacun Coordinated alert and information sharing circuit based on each individual's missions and skills
Centré sur la personne et son entourage Focused on the person and his or her relatives family or caregivers

Source : document interne réalisé par le réseau Gérard Cuny et la mission santé bien-être de la Métropole du Grand Nancy. Reproduction autorisée par le réseau Gérard Cuny et la Mission Santé Bien-Être de la Métropole du Grand Nancy.

Enfin, dans le cadre du Contrat Local de Santé intercommunal 2019-2023, la Métropole du Grand Nancy s'est donnée pour mission d'améliorer l'information, la communication et la prévention en matière de santé, notamment en luttant contre les infox ou fake news. À travers son rôle de médiation et d'explication à l'égard de la population, le but de cette mission est de sensibiliser à la vérification des sources, d'orienter vers des sites fiables et reconnus, et de diffuser les préconisations de la cellule d'experts mise en place par la Métropole. Cette mission, lancée avant le début de l'épidémie, joue un rôle majeur dans le cadre de la crise de Covid-19, en écho aux propos de Marianne Kedia (2016). Elle nous rappelle que le risque de développer un état de stress post-traumatique est significativement accentué par l'exposition aux médias. Ainsi, divers auteurs préconisent une sensibilisation aux médias et à la recherche d'informations comme un moyen efficace pour lutter contre l'anxiété. 


\section{Sur la mobilité, les transports et l'espace public} communes à travers le Plan Local d'Urbanisme Intercommunal (PLUI) qui comprend le plan de déplacements urbains (DPU). Dans ce cadre, il a été décidé de rendre l'ensemble $\mathrm{du}$ réseau des transports en commun gratuit pour le personnel soignant et de réserver des navettes aux personnels travaillant dans les centres hospitaliers pendant la période de pandémie. mise en place du plan vélo, avec une expérimentation de pistes cyclables supplémentaires, une facilitation de la mise à disposition de vélos ainsi qu'une politique d'encouragement à son usage. La Métropole est également amenée à repenser et redéfinir ses choix d'investissements en termes d'infrastructures urbaines, notamment un déploiement des pistes cyclables définitives et des zones piétonnes, dans l'objectif de préserver sa « santé urbaine » comme suggéré par l'urbaniste new yorkais Jens Aert (Courrier International, 2020). qui devrait être repensé à l'image de la modification de notre manière de partager l'espace public. Pour exemple, la largeur des trottoirs ne permet pas un respect optimal des distances de sécurité sociale. Pour autant, la Métropole, en collaboration avec les citoyens et tout autre acteur, souhaite pouvoir faire preuve de créativité et d'innovation afin d'éviter de déshumaniser ces espaces publics.

\section{Les actions concrètes menées par la Métropole pour préparer la sortie du confinement}

À ce jour, les experts constituant la cellule ont été sollicités à plus de 40 reprises pour des demandes concernant prioritairement le champ de l'accompagnement en santé mentale et le champ de la logistique. Ainsi, la collectivité a soutenu et mis en place des aides allant du soutien financier et logistique, à la mise en place de plateformes d'écoute médico-psychologiques. Le Contrat Local de Santé intercommunal de deuxième génération 2019-2023 a permis d'identifier la Métropole comme un levier incontournable dans le champ de la santé ; notamment dans le cadre de la gestion de crise. Ainsi elle a pu être sollicitée par ses partenaires pour mener à bien, notamment à travers le dispositif " prévention conséquences confinement ", diverses actions visant à accompagner chaque citoyen pendant cette période de crise et au-delà, et plus particulièrement les personnes vulnérables et/ou isolées qui présentent un risque plus élevé de développer une forme grave de Covid-19 et dont l'isolement peut entraîner des vulnérabilités supplémentaires. En effet, selon la synthèse nationale de la Fédération Nationale des Observatoires Régionaux de la Santé (FNORS) datée de mai 2020, plus de 36,8\% des individus âgés de plus de 75 ans dans le Grand Est vivaient seuls en 2016 (cf.

Revue francophone sur la santé et les territoires , Pandémie, crises et perspectives : lectures territoriales de la Covid-19 
figure 5). De même, selon la FNORS, plus de 70,6\% des personnes bénéficiaires de l'allocation adulte handicapé (AAH) vivaient seules dans le Grand Est, en 2016. Ces chiffres soulèvent la part importante de personnes vulnérables vivant seules sur le territoire et pour lesquelles le dispositif « prévention conséquences confinement » peut s'appliquer.

Figure 5. Personnes de 75 ans et plus vivant seules (pour $100-2016$ )

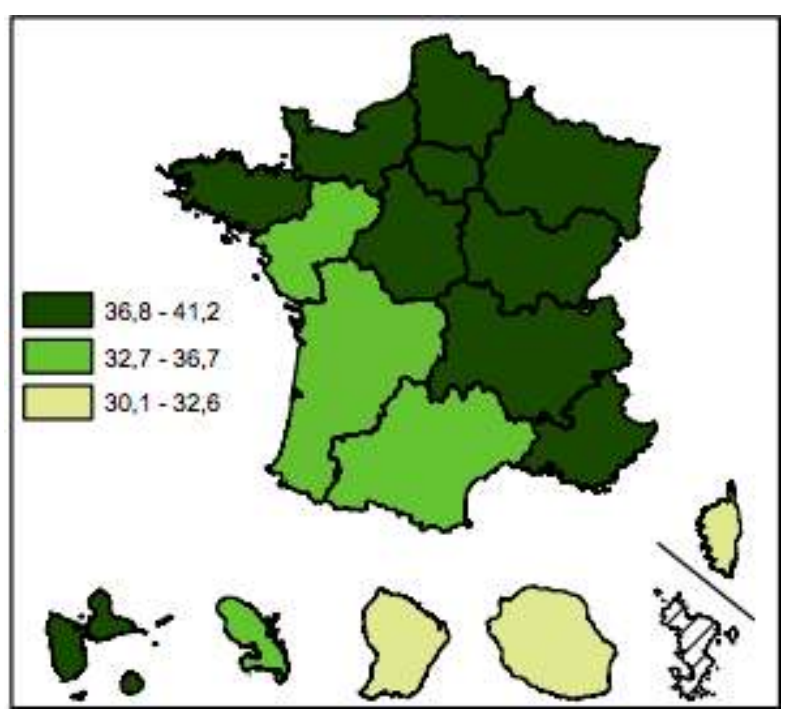

Source : Fédération nationale des observatoires régionaux de santé. (2020). Appui au déconfinement Profils de territoires. Synthèse nationale. Consultable en ligne : https://www.scoresante.org/ uploadedFiles/SCORE-Sante/Profils/Fnors_Profils_Deconfinement_SyntheseNationale_2020.pdf

Cette cellule de réflexion après confinement est toujours active et les experts continuent de se réunir de façon ponctuelle en visioconférence pour échanger sur leurs observations, ouvrir le débat, réaliser des points de situation et d'éventuels ajustements, continuer de formuler des recommandations. La dernière rencontre, en date du 24 juin 2020, a permis de soulever une absence de communication, notamment au niveau de l'hygiène et les partenaires ont soulevé l'intérêt de recourir au Contrat Local de Santé pour instaurer des actions de proximité et de prévention dans ce domaine.

Cette période de crise a permis de faire ressortir les fragilités et les insuffisances, mais également la capacité de résilience et de collaboration locale des acteurs publics et privés, ainsi que la coexistence compatible entre l'action institutionnelle et les initiatives citoyennes. La Métropole souhaite désormais mettre en avant et consolider les actions de solidarité qui ont su émerger en cette période de difficulté. Dans ce cadre, les experts de la cellule CRAC ont soumis l'idée d'instaurer des ateliers "santé ville " dans les quartiers dont l'objectif ne serait pas uniquement de construire pour les gens, mais de construire avec les gens. En effet, s'il s'avère qu'il existe des disparités au niveau sociodémographique et économique face à la santé, dans certains quartiers de la Métropole, notamment les QPV (cf. figure 6), il s'avère également que ces derniers n'ont pas été impactés par l'épidémie de manière plus conséquente que les autres. 


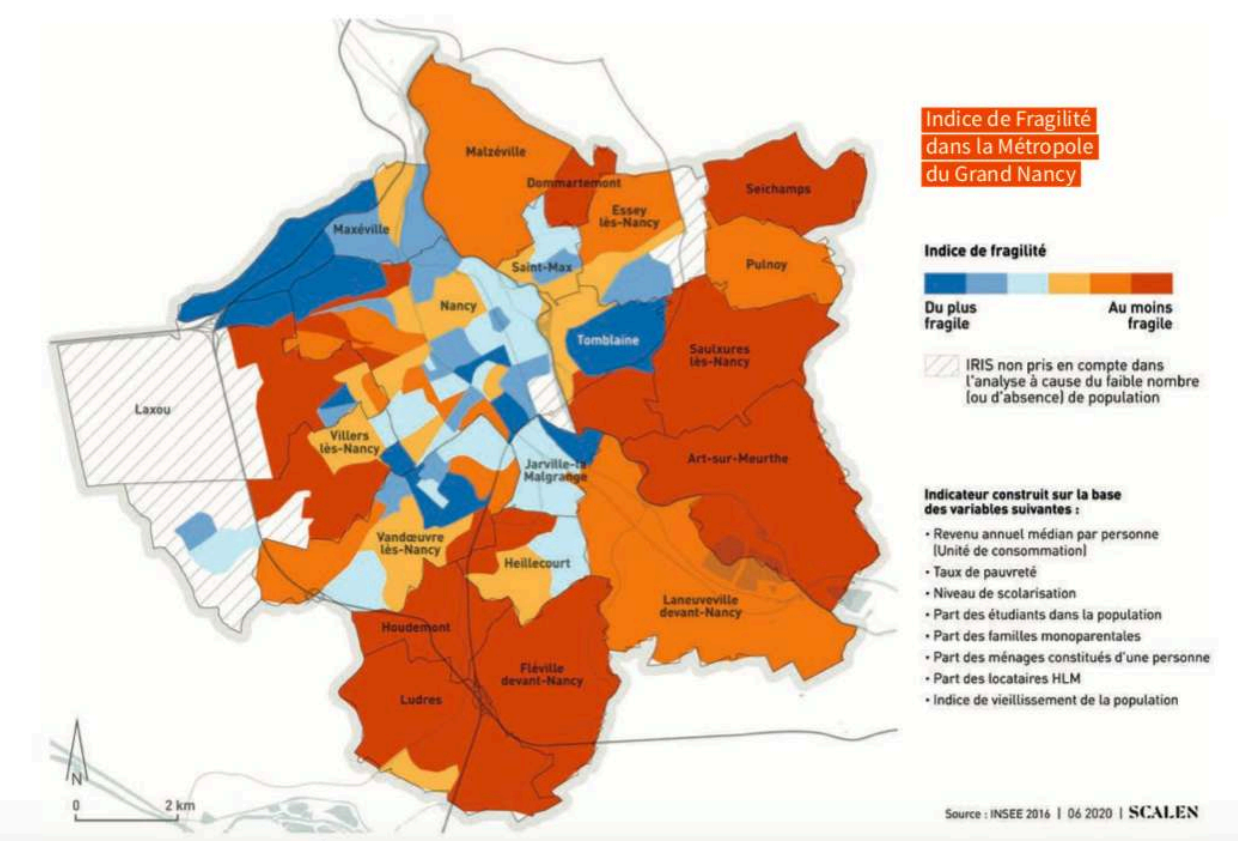

Source : Agence SCALEN. (2020). Atlas Santé Bien-Être de la Métropole du Grand Nancy. Consultable en ligne : https://www.grandnancy.eu/fileadmin/fichiers/VIVRE_ET_HABITER/Sante/Atlas_Sante.pdf

Les experts ont également émis l'idée de pouvoir instaurer des actions sur le long terme. De même, si la cellule CRAC se concentre aujourd'hui sur les dimensions sanitaires de la crise, elle pourrait susciter, au-delà, des réflexions connexes sur les fragilités et la capacité de résilience de la société métropolitaine.

Enfin, dans le cadre du Contrat Local de Santé intercommunal 2019-2023, la Métropole du Grand Nancy a mis en place, en 2020, un observatoire métropolitain Santé et Bienêtre, avec l'appui de l'agence SCALEN, l'agence de développement des territoires Nancy Sud Lorraine. L'objectif est de développer un outil permettant d'orienter les politiques publiques de santé. Dans un premier temps, un atlas de l'état zéro a été constitué à partir des données produites par divers acteurs locaux, régionaux et nationaux. Par la suite, ces données seront cartographiées à l'échelle du territoire dans le but d'analyser les phénomènes à l'œuvre et d'améliorer leur compréhension à l'aide de données comparatives observées à d'autres échelles géographiques. La constitution de cet Atlas a permis d'identifier les populations vulnérables sur le périmètre métropolitain lors de cette crise.

\section{Après la crise, la résilience}

Après une première phase dédiée aux urgences sanitaires, nous percevons le fait que cette crise génère également des implications fortes sociales, sociétales, et économiques qu'il faudra appréhender dans leur ensemble et dans la durée.

En ce sens la Métropole et les communes ont à préparer de manière collective et concertée la " convalescence sociale et sociétale » qui sera longue. C'est l'objet du plan de résilience métropolitaine, à vocation très opérationnelle.

Il s'inscrit pleinement dans l'esprit du projet métropolitain (consultable en ligne sur le site de la Métropole du Grand Nancy). Ainsi la santé et le bien-être sont les liens qui 
caractérisent culturellement un territoire déjà passé par d'autres épreuves économiques ou sociales dans son histoire. La cohésion des acteurs publics et le développement des nouveaux modes de faire constituent des défis contemporains permanents. Pour ce faire, les principes de mise en œuvre qui structurent le plan de résilience sont les suivants : être efficace ensemble, prolonger ou réinventer lorsque nécessaire les processus d'horizontalité, de coopération et d'intelligence collective, conforter et promouvoir les initiatives citoyennes, développer la connaissance et la partager. Ces principes s'appuient sur la notion de résilience qui représente la capacité à «continuer à se projeter dans l'avenir en dépit d'évènements déstabilisants, de conditions de vie difficiles, de traumatismes sévères " (Manciaux, Lecomte et Cyrulnik, 2001), mais également à remobiliser ses forces pour s'adapter, rebondir et se réinventer (Molenda, CN2R, 2020).

Il s'agira que les acteurs publics et institutionnels, opérateurs, acteurs économiques, sociaux, associatifs, culturels travaillent ensemble. Il semble pertinent et nécessaire de coopérer et co-construire pour développer et consolider un territoire résilient pour la gestion de cette présente crise et dans la perspective d'autres crises potentielles, sanitaires, environnementales ou climatiques ; un territoire plus apte dès maintenant, et pour l'avenir, à absorber les chocs susceptibles de fragiliser la société au-delà des dimensions infrastructurelles, et à rebondir dans les meilleures conditions possibles.

Cette résilience recherchée est multidimensionnelle ; la Métropole propose de l'investir autour de six grands enjeux, déclinés en une quarantaine d'actions, prioritaires pour la population et pour les acteurs du territoire : l'accompagnement de la crise sanitaire, la cohésion de la société métropolitaine, l'adaptation du travail et des activités économiques, les dimensions éducatives /culturelles / sportives et de loisirs, le partage équilibré des espaces publics, leviers de mobilité, mais également de liens sociaux, culturels, et d'activités commerciales, et enfin les mutations économiques et écologiques qui restent à poursuivre dans la continuité des transitions engagées.

Ce plan de résilience métropolitaine constitue un cadre évolutif et ouvert. La démarche elle-même consistera à soulever des questions, et à les traiter au fur et mesure de la clarification des contextes, avec humilité et persévérance.

\section{Conclusion}

Cette étude présente un tout premier retour d'expérience opérationnel sur les actions menées sur le territoire métropolitain dans un contexte tendu de crise sanitaire. Cet écrit a permis de mettre en lumière le levier que constitue le Contrat Local de Santé en matière d'organisation territoriale de la santé. Depuis 2013, dans le cadre du CLS de première génération 2013-2017, la Métropole du Grand Nancy a engagé des habitudes de travail en commun avec ses partenaires, notamment l'ARS et la Préfecture de Meurthe-et-Moselle, mais également les acteurs du domaine associatif qui ont pu adapter leurs actions aux besoins du moment, comme le démontre la mise en place du dispositif "Prévention Conséquences Confinement». Ainsi, ce contrat a permis à la Métropole, au fil de la crise sanitaire de la COVID19, une agilité certaine au côté des acteurs de terrain que sont les professionnels du soin, de la santé, du bien-être, et en particulier les porteurs d'actions du Contrat Local de Santé (cf. figure 7). La figure 7 présente une proposition d'organisation territoriale métropolitaine en cas de crise. Dans le cadre de la mise en œuvre, au niveau national ou local, d'une gestion de crise, la 
communication de crise, les décisions prises et les mesures mises en œuvre doivent faire partie intégrante du dispositif de gestion de la crise. A cet effet, un plan de communication spécifique identifiant notamment la stratégie de circulation de l'information à mettre en œuvre, ainsi que les outils à disposition des autorités publiques, les relais de l'information potentielle (professionnels de santé, structures associatives...) ainsi que les modalités de leur mobilisation doivent être intégrés dans un schéma d'ensemble. La répartition des rôles de chacun devra faire l'objet d'une réflexion pour conduire de manière efficiente la gestion de crise.

La cellule de crise créée spécifiquement dans le cadre de la Covid-19 s'inscrit dans l'organisation illustrée par la figure ci-après. Cette organisation tend à optimiser la communication des informations entre les divers partenaires concernés par la gestion de cette crise. La Métropole du Grand Nancy dispose, par ce biais, d'un engagement et d'un devoir de conseils envers ses concitoyens, notamment dans le domaine de la promotion de la santé.

Figure 7. Proposition d'organisation territoriale du système de santé de la Métropole du Grand Nancy en période de Covid-19

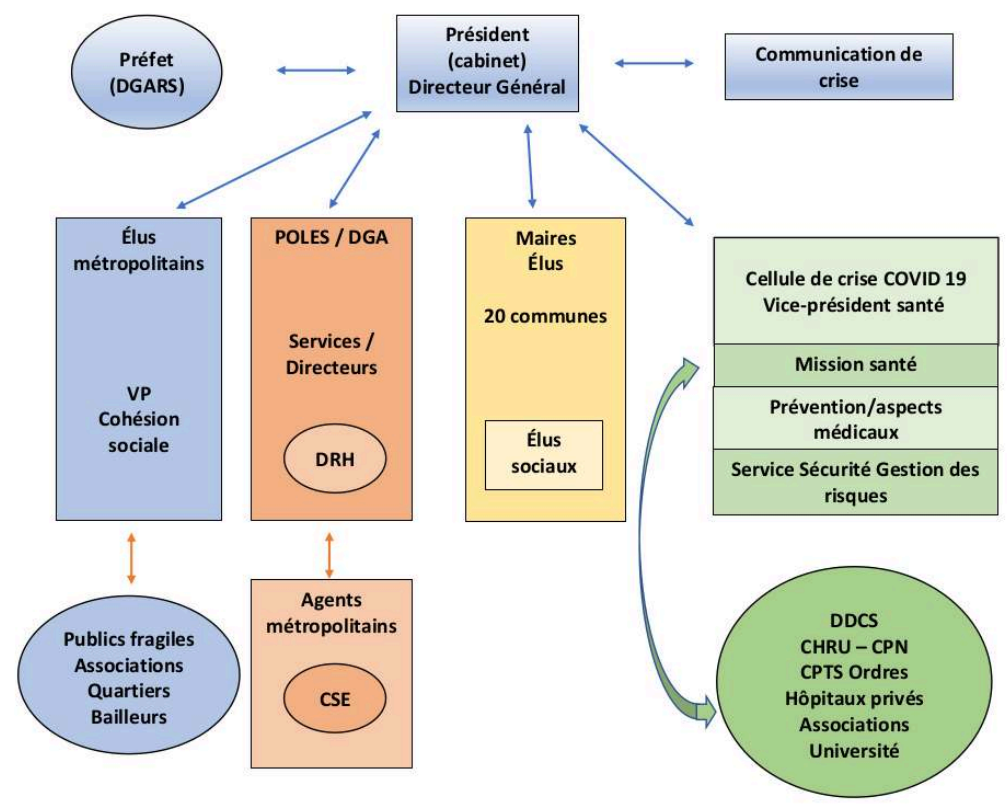

Légende : DGARS (Direction Générale de l'Agence Régionale de Santé), DDCS (Direction Départementale de la Cohésion Sociale), VP (vice-président), CPN (Centre Psychothérapique de Nancy), CPTS (Communauté Professionnelle Territoriale de Santé).

Source : document interne à la Mission Santé Bien-Être non publié. Reproduction autorisée par la mission santé bien-être de la Métropole du Grand Nancy.

Dans le cadre du Contrat Local de Santé 2019-2023, la CPTS mène une enquête sur le respect des gestes barrière en population générale. Cette enquête vise à mettre en évidence les freins au changement et à identifier les populations les moins aptes à les appliquer. Les informations recueillies permettront d'adapter les communications et de former des ambassadeurs de santé volontaires issus des associations composant le Contrat Local de Santé de la Métropole du Grand Nancy. 


\section{Articles scientifiques}

Anaut, M. 2005. Le concept de résilience et ses applications cliniques. Recherche en soins infirmiers, 82(3), 4-11. https://doi.org/10.3917/rsi.082.0004>

Brooks, S. K., Webster, R. K., Smith, L.E., Woodland, L., et al. 2020. The psychological impact of quarantine and how to reduce it: rapid review of the evidence. The Lancet, 395, 912-920. https:// doi.org/10.1016/S0140-6736(20)30460-8

Campanella, T.J. 2006. Urban resilience and the recovery of New Orleans. Journal of the American Planning Association, 72(2). https://doi.org/10.1080/01944360608976734

Dumont, G. 2020. Covid-19 : la fin de la géographie de l'hypermobilité ? Les analyses de Population \& Avenir, 29, 1-13. https://doi.org/10.3917/lap.029.0001

Lhomme, S., Serre, D., Diab, Y. et Laganier, R. 2010. Les réseaux techniques face aux inondations ou comment définir des indicateurs de performance de ces réseaux pour évaluer la résilience urbaine. Bulletin de l'association des géographes français, 487-502. https://www.persee.fr/doc/ bagf_0004-5322_2010_num_87_4_8193>

Manciaux, M. et al. 2001. La résilience : résister et se construire. Cahiers Médicaux Sociaux.

Tissandier, P., Mariani-Rousset, S. 2019. Les bénéfices du télétravail. Mobilité modérée : réduction du stress et des émissions de gaz à effets de serre. RFST. https://journals.openedition.org/rfst/ 397

Toubin, M., Lhomme, S., Diab, Y. Serre, D., Laganier, R. 2012. La résilience urbaine : un nouveau concept opérationnel vecteur de durabilité urbaine ? Développement durable \& Territoire, 3(1). https://doi.org/10.4000/developpementdurable.9208

\section{Articles de presses}

Chignard, A. 2020. Comment bien manager le retour au travail après le confinement. https:// www.psychologies.com/Actualites/Vie-pro/Comment-bien-manager-le-retour-au-travail-apresle-confinement

Shenker, J., 2020, Changer la ville. Courrier International, 1541, 6-8.

\section{Littérature non scientifique}

Center for the Study of Traumatic Stress. 2020. Effets psychologiques de la quarantaine pendant l'épidémie de coronavirus : ce que les professionnels de santé doivent savoir. Traduit par Laetitia Jamet.

Kedia, M. 2016. Attentat de Nice : « les chaînes d'info fonctionnent comme un cerveau traumatisé ». https://www.telerama.fr/television/lors-des-attentats-les-chaines-d-infofonctionnent-comme-un-cerveau-traumatise-marianne-kedia-psychologue,140140.php 
Lafond, M. 2020. Petit guide pratique du confiné : Prévention des risques psychologiques dans une période de confinement. https://sgen-cfdt.fr/contenu/uploads/sites/29/2020/03/PetitGuide-pratique-du-Confin\%C3\%A9-sans-audio.pdf

Lemogne, C., Dantchev, N.N Abgrall, G. 2020. Principes du soutien psychologique des professionnels de santé en situation de crise sanitaire. AP-HP, Centre Université de Paris. http:// www.cphg.org/wp-content/uploads/2020/04/Soutien-psychologique-aux-professionnels-desante.pdf

Molenda, S. 2020. Covid-19 : Renforçons notre résilience. Centre National de Ressources et de Résilience, France. http://cn2r.fr/wp-content/uploads/2020/03/Renforcons-notre-resilience.pdf

Tordjman, S., Schröder, C., Delorme, R. 2020. Du confinement au déconfinement : nouvelles perspectives en pédopsychiatrie. https://www.afpbn.org/wp-content/uploads/2020/05/ DuConfinementauDeconfinement.pdf

\section{Rapports officiels}

Avis $n^{\circ} 6$ du Conseil scientifique COVID-19. 2020. Sortie progressive de confinement, prérequis et mesures phares. https://solidarites-sante.gouv.fr/IMG/pdf/ avis_conseil_scientifique_20_avril_2020.pdf

Fédération nationale des observatoires régionaux de la santé. 2020. Appui au déconfinement, profils de territoires : synthèse nationale. https://www.scoresante.org/uploadedFiles/SCORESante/Profils/Fnors_Profils_Deconfinement_SyntheseNationale_2020.pdf

HAS. 2020. Impact de l'épidémie de Covid-19 dans les champs social et médico-social. https:// www.has-sante.fr/upload/docs/application/pdf/ 2020-04/2020_04_21_contribution_csms_covid19.pdf

HAS. 2020. Prise en charge des patients souffrant de pathologies psychiatriques en situation de confinement à leur domicile. Réponses rapides dans le cadre du Covid-19. https://www.hassante.fr/jcms/p_3168631/fr/prise-en-charge-des-patients-souffrant-de-pathologiespsychiatriques-en-situation-de-confinement-a-leur-domicile

Jeantit, F., Keita, M., Cailler, J., Danan, J-L., Demange, M. 2020. Atlas Santé Bien-Être de la Métropole du Grand Nancy 2020. Scalen. https://www.grandnancy.eu/fileadmin/fichiers/ VIVRE_ET_HABITER/Sante/Atlas_Sante.pdf

OMS. 1993. Classification internationale des troubles mentaux et des troubles du comportement (CIM 10)

OMS. 2012. Les premiers secours psychologiques : Guide pour les acteurs de terrain. https:// apps.who.int/iris/bitstream/handle/ 10665/44779/9789242548204_fre.pdf;jsessionid=7F2E506215074F9E60CBC9D330228A7A? sequence $=1$

OMS. 2020. Mental health and psychosocial considerations during the COVID-19 outbreak. https://www.who.int/docs/default-source/coronaviruse/mental-health-considerations.pdf 


\section{Documents internes}

Agence de développement des territoires Nancy Sud Lorraine. 2020. Atlas Santé Bien-Être de la Métropole du Grand Nancy 2020. https://www.grandnancy.eu/fileadmin/fichiers/ VIVRE_ET_HABITER/Sante/Atlas_Sante.pdf

Métropole du Grand Nancy. 2015. Contrat de ville du Grand Nancy 2015-2020. https:// www.grandnancy.eu/fileadmin/fichiers/LA_METROPOLE/Democratie_participative/ 2018_06_12_contrat_de_ville.pdf

Métropole du Grand Nancy. 2017. Contrat de ville 2015 - 2020, Rapport annuel 2017. https:// www.grandnancy.eu/fileadmin/fichiers/LA_METROPOLE/Democratie_participative/ MGN_rapport_CV-2019.pdf

Métropole du Grand Nancy. 2019. Contrat Local de Santé 2è génération 2019/2023. https:// www.grandnancy.eu/fileadmin/fichiers/VIVRE_ET_HABITER/Sante/CLS_2019-2023.pdf

\section{RÉSUMÉS}

Cette étude concerne les enjeux sociaux et spatiaux de la gestion de la pandémie, et notamment la préparation à la sortie du confinement sur le territoire de la Métropole du Grand Nancy. Face aux risques sanitaires liés à la Covid-19, la Métropole a mis en place des mesures afin de doter l'ensemble de ses 20 communes de ressources pour préparer au mieux cette sortie du confinement, ainsi que la convalescence de la société grâce à une approche globale de la résilience. L'objectif visé est l'accompagnement des citoyens de manière complémentaire au cadre fixé par l'État. La Métropole a sollicité ses partenaires afin de constituer une cellule de réflexion. Cette cellule pluridisciplinaire de réflexion avant sortie de confinement continue d'aider les représentants des communes à gérer cette situation inédite grâce à un système de communication visant à apporter des réponses concrètes aux problématiques rencontrées. L'ensemble de ces travaux s'est concrétisé sous la forme d'un plan de résilience métropolitaine, à vocation très opérationnelle.

\section{INDEX}

Mots-clés : gestion de la pandémie, sortie de confinement, résilience individuelle, résilience urbaine, cohésion sociale

Index géographique : Nancy

\section{AUTEURS}

\section{CHARLOTTE COPPLET}

Étudiante en psychologie clinique et légale, France, Université de Lorraine

\section{MARTINE BATT}

Professeur de psychologie légale et psychopathologie, France, Université de Lorraine, Responsable axe GRC, laboratoire INTERPSY 4432 


\section{ANDRÉ ROSSINOT}

Docteur en médecine, ancien président de la Métropole du Grand Nancy, France.

\section{JANE-LAURE DANAN}

Docteur en sciences de la vie et de la santé, Responsable de la Mission Santé et Bien-Être. France, Métropole du Grand Nancy, Université de Lorraine, chercheur associé Axe PRISME, laboratoire INTERPSY 4432 